\title{
Experiences Managing and Maintaining a Collection of Interactive Office Door Displays
}

\author{
Dan Fitton \\ +44 (0) 1524594532 \\ df@comp.lancs.ac.uk
}

\author{
Keith Cheverst \\ +44 (0) 1524594539 \\ kc@comp.lancs.ac.uk
}

\begin{abstract}
To date there have been very few ubicomp systems that have been deployed and evaluated 'in place' and over the longer term. Consequently, the issues related to the management and maintenance of such systems remain a very much under explored (but nonthe-less critical) area. This paper discusses our experiences managing and maintaining the Hermes system, which provides asynchronous messaging services for users and runs on multiple public display appliances situated outside offices in the computing department of Lancaster University. Hermes has been running $24 / 7$ for over 15 months, and receives regular use by a range of users.
\end{abstract}

\section{Keywords}

Ubicomp Deployment, Situated Display Appliance, Management Issues, Wireless Networking, Interactive Systems.

\section{INTRODUCTION}

Weiser's vision of ubiquitous computing [9]; moving technology away from the focus of a 'single box' and 'into the fabric of everyday life', seems to imply the need for highly-available situated technology. Today there are many examples of mobile devices allowing users access to applications and services wherever they go, however these simply allow us to take the 'box' with us. One of the key requirements to providing a real ubiquitous computing environment is situating technology in a range places.

This paper presents experiences of managing and maintaining the Hermes system [1], a system of deployed interactive office door displays, providing asynchronous messaging services for users in the computing department of Lancaster University. The displays are small PDA based devices situated outside offices and accessible to all passers-by. One of our overriding aims has been to deploy and evolve Hermes in the longer term. This is to investigate the interactions that (do and do not) occur, observing how the system is used on a day-to-day basis. Through our approach we hope to see how situated technology can be put to use in everyday scenarios, and what the principal factors in design and development on such systems are.
Such a system deployed and used on a day to day basis clearly requires some sort of management element to help it run smoothly. This paper presents the additional management agent that we have developed for maintaining the 'smooth' running of the Hermes system, and in particular to address some of major technical problems we have encountered during the last 15 months of use.

The following section of this paper ('The Hermes System') presents an overview of the Hermes project and the third section ('Design of the Hermes System') presents some of the key design issues behind Hermes. The fourth section ('Experiences of Long Term Deployment') then introduces some of the motivating factors for supporting a management element within Hermes. Following this the fifth section ('Supporting and Managing Hermes') presents an overview of the key functionality supported by our implemented and deployed management agent. This is followed by short overview of related work in the area of office door displays and a future work section. Finally some concluding remarks are presented in the last section.

\section{THE HERMES SYSTEM \\ Overview}

One of our goals for developing and deploying the Hermes system was to explore whether the traditional way of leaving messages on Post-it ${ }^{\mathrm{TM}}$ notes in 'semiprivate' places, such as office doors, could be enhanced with a digital equivalent. In order to explore this area we have designed and deployed a digital asynchronous messaging system (named Hermes after the messenger to the gods in Greek mythology) within the main computing building at Lancaster University.

The Hermes system supports remote interaction through a web portal and by allowing messages to be created using a mobile phone via SMS (Short Message Service) [2].

Devolvement work started on the Hermes system in October 2001 and the first unit was installed outside one of the offices in the computing department in March 2002. The system comprises a central server and a number of wall mounted units (referred to as Hermes displays). 
Figure 1 illustrates the first Hermes display to be deployed in the department.

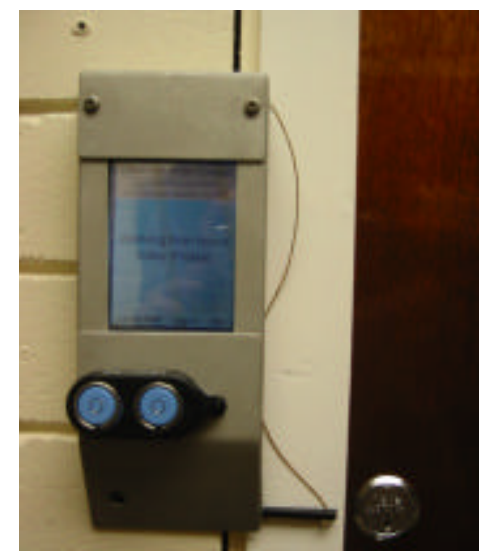

Figure 1: Example of an early Hermes display.

The design of Hermes displays was required to meet a number of installation requirements.

\section{Requirements}

The deployment of the Hermes system was subject to three main requirements, namely: units should comply with university health and safety regulations, units should comply with disabilities legislation, units should be both straightforward to deploy and units should be relatively secure.

Current U.K. disabilities legislation states that public facilities need to be positioned at a height that does not unduly discriminate against people using a wheelchair. For this reason, it was necessary to place the units at a fairly low height (approximately $150 \mathrm{~cm}$ ) off the floor. However, one of the implications of this is that the device is quite awkward to use for taller people. This problem highlights an interesting variation on the theme of private ownership vs. public use.

One of our key requirements for Hermes displays was that they should be very easy to deploy. We felt that this requirement would be best met by designing the Hermes display as a self contained unit. We also wanted the display to be relatively easy to develop and so we chose to adopt a PDA based solution. We had hoped that the use of wireless communications would mean that cabling would not be required from a Hermes display to its associated office. Unfortunately, however, the battery life of a PDA is still relatively short and so it has proved necessary to take (LV) power from offices through a small drilled hole in the door mounting.

Although access to the department is restricted during evenings and weekends the department has unrestricted access at all other times. For this reason, we needed to mount displays in such a way that opportunistic theft of the device would be difficult. The case for the displays was also required to restrict access to the buttons on the PDA device. This is to prevent malicious or accidental termination of the application.

\section{Functionality}

Our design approach has been implement a small number of features well, rather than attempting to provide every feature technically feasible. This enables us to provide the levels of ease of use and dependability usually found with information appliances.

The functionality supported by the system can be considered from two main perspectives: the perspective of the owner of the Hermes display and the perspective of a visitor to the Hermes display.

\section{Functionality available to the Owner}

The system provides the owner of the Hermes display with two key functions: the ability to create a message to appear on the display, and, the ability to read messages left by visitors.

Typically, the owner will create a message to appear on their Hermes display by entering some appropriate text using the web interface shown in figure 2 . The web interface can also be used to upload a graphical image for display, such as an animated GIF.

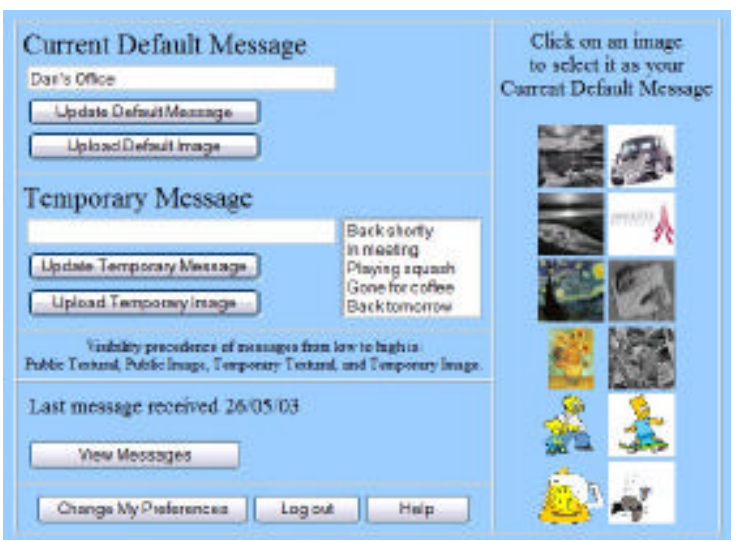

Figure 2: The Hermes Web Interface.

Initially, only the web interface could be used by the owner of the Hermes display in order to create messages. However, after a short period of use it became clear that it is often only when closing the office door that one thinks to leave a message. For this reason we added a feature to enable the owner to create a freehand message by using an interface on the door display itself. This process does, however, require the owner to authenticate themselves with the system; this would typically be achieved by the owner docking his or her iButton [3] or entering a username/password via a simple GUI on the Hermes display.

The owner can read his or her messages remotely via a web browser (see figure 3). 


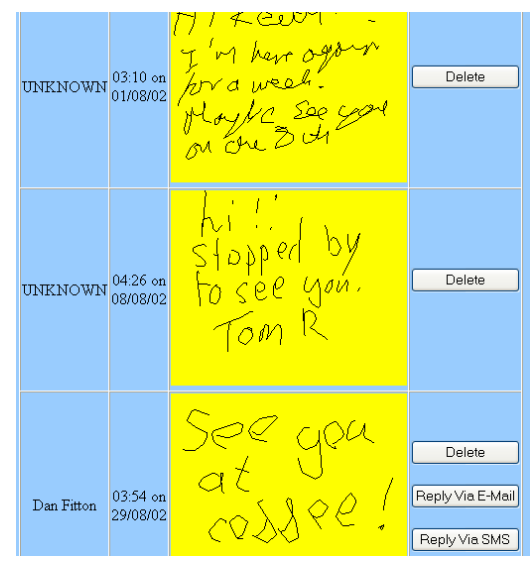

Figure 3: Viewing Messages via Hermes Web Portal.

\section{Functionality available to the Visitor}

In the current implementation, a visitor must be colocated with a Hermes display in order to leave a message. The user simply has to tap on a 'leave note' button on the Hermes display and then use the attached pen to 'scribble' a message on the touch sensitive display. Once the user taps on the 'finished' button the display on the unit is updated to reflect the fact that the owner has an additional message waiting to be read.

A visitor is not permitted to read the messages left by other visitors for the owner of a Hermes display, providing a privacy advantage over the traditional Post-it ${ }^{\mathrm{TM}}$ note. Authentication by a visitor allows them to remotely view any messages left on their own Hermes display.

\section{DESIGN OF THE HERMES SYSTEM \\ Design of the Hermes Display Hardware}

Having decided to develop a system using an off-theshelf PDA, we considered various candidates. After consideration we decided upon using the Jornada 568 for two main reasons. Firstly, the unit has a built-in compact-flash type 1 slot so does not need an additional expansion jacket. Secondly, the unit has a relatively square shape and we felt that this would simplify case design. Initially all Hermes displays were equipped with a compact flash type 1 802.11b wireless network interface.

In order to allow the units to be deployed securely, a case was designed to hold the unit. The case is made from aluminium, and can accommodate an iButton reader. This is wired to the serial port of the Jornada in order to support authentication.

\section{Software}

From an early stage we decided to use a Java for development. However, finding a Java virtual machine (VM) that met our requirements was a difficult process, particularly one that supported Java COMM API (to support a serial iButton reader). Eventually, we chose to adopt NSlcoms CrEme [7] version 3.2 running on the PocketPC2002 operating system as our development platform.

\section{Overall System Architecture}

The overall system architecture of the Hermes system is shown in Figure 4. The large oval represents the typical entities associated with a given user.

At the heart of the system is a single central server application written in Java 2 running under Linux. It provides the following key functions:

- centralized storage for messages and user profile information,

- communication with the SMS Gateway,

- hosting of the web portal.

As illustrated in figure 4, the system utilizes both wireless (802.11b) and wired Ethernet network infrastructures. In order to support the reception of SMS messages, the central server communicates with a Wavecom DB02 GSM terminal over a serial link.

Messages left by visitors on Hermes displays are encoded using run-length encoding before being sent to the server, where they are re-encoded as GIF files. These file are then made available using an Apache web server.

The web portal is implemented using Java servlets running on a Jakarta Tomcat servlet runner. This enables the dynamical generation and publication of html web pages (see figure 3) using the Java language and straightforward integration with other components using Java RMI.

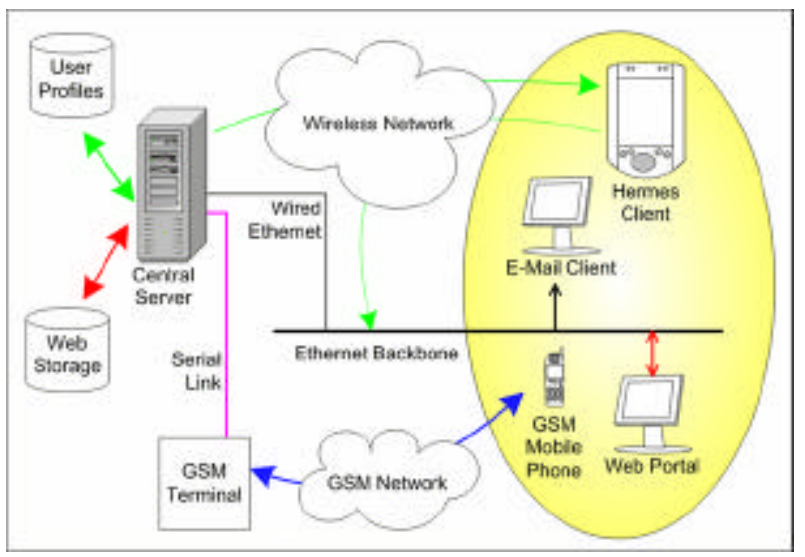

Figure 4: The System Architecture of Hermes.

\section{Current Deployment}

At the time of writing we have 10 units deployed on one floor of the Computing Department at Lancaster. The majority of units are situated outside the offices of computer scientists. However, in order to avoid including only 'techies' in our study, two of the units are situated outside the offices of departmental secretaries, and another outside the office of a sociologist (and certainly not a techie). 


\section{EXPERIENCES OF LONG TERM DEPLOYMENT}

To investigate the use of Hermes over the long term obviously requires regular use during that time. This is perhaps one of the more demanding issues inherent to this system, and where it contrasts with most comparable projects, which have tended to produce few 'end systems' and where not deployed widely or over long periods (examples include [5], [4] and [6]).

We require users to integrate the Hermes system into their current patterns of use, for this to happen there will always be an initial cost-benefit trade off for users: It generally seems to take adaptation, consideration and time to integrate new technology into a daily routine. When designing Hermes the basic functionality we attempted to provide was that of paper Post-it $\circledast$ notes placed on office doors \& message 'whirlers' as shown in figure 5.

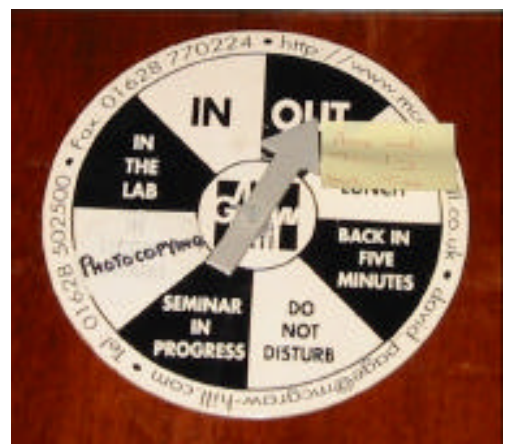

Figure 5: Typical message 'whirler'

Augmenting an existing system proves advantageous as there is always a backup system for users to rely on if there is a temporary fault with Hermes. However, the problem is ensuring that people use Hermes instead of these traditional systems. One of the most disappointing scenarios we have encountered is users placing Post-it $\AA$ notes next to working Hermes displays.

From our research it seems that there are two important factors to encourage and maintain use of Hermes, ease of use and trust. We found that there is a limit to how much effort a task requires before it is unfeasible as part of a daily routine. This limit proved to be lower than originally thought, and we have continually improved ease of use though the addition of various new software features.

The issue of trust seems to be linked directly to dependability, users will only bother to use a new system, let alone as part of their daily patterns, if they think it works. This seems a logical requirement from a user's point of view, but essentially translates to very high levels of reliability. This is difficult on a system such as Hermes, especially given the current hardware and software platforms.

Logging the use of Hermes
All aspects of the Hermes system are logged continually. This ranges from the actions generated by UI components on Hermes displays to messages shared, sent and received by users. All information that we wish to log is sent to the central server to be recorded. We maintain this level of logging as it is hard to pre-empt what information may need to extract from the logs in the future. Recently we need to investigate the level and type of context sharing taking place through Hermes by its users. Extraction of messages shared by users from the logs proved straightforward, though we had no mechanism in place for classifying the context shared. Extracting this information involved tagging each message (of which there were over 300) by hand using the categories of activity, temporal and location (a small snippet of the $\log$ is shown in figure 6).

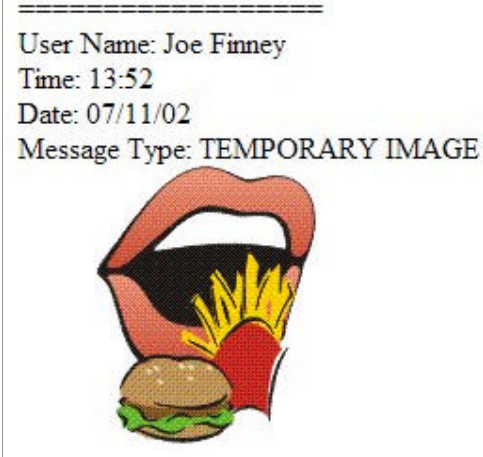

口UT TD LUNCH!

Context: ACTIVITY

User Name: Keith Cheverst

Time: 16.54

Date: $07 / 11 / 02$

Message Type: TEMPORARY TEXTURAL

Message: Out to lunch - back at 2.

Context: TEMPORAL ACTIVITY

Figure 6: Information extracted from Hermes logs

\section{Technical Problems Encountered}

Many of the initial problems after deploying Hermes displays seemed to be attributed to the Java VM used, drivers for the various wireless network cards, and the PocketPC2002 operating system. Individually or together, running continually, these elements have proved a source of instability, occasionally crashing the Hermes displays. These crashes seem to have been reduced with updated versions of the Java VM, device drivers for the wireless network cards and PocketPC2002 updates. After initial testing is was intended to rivet the tops onto the cases for security, though to-date we have not felt confident enough in the stability of the devices, so for the moment the tops are screwed on. A current solution to this problem is to route the reset switch on the PDA device to inside the office of the owner. 
A second source of problems is the experimental wireless network used by the Hermes displays. The major problem is that of signal strength. The displays can be thought of as 'thin' clients, little information is stored at them (due to their limited storage, small processing capacity and relative unreliability), and the majority of information is stored at the central server. Because of this most user interface actions (such as logging in, leaving a message etc) on the Hermes displays require communication, via Java RMI, with the server. We have found that RMI calls using our present Java VM seem very sensitive to network quality, and consume large amounts of CPU time. This means that if a user attempts to perform an action requiring communication with the server, and there is a temporary degradation in wireless signal strength (for example due to users standing in front of a Hermes display), the application may halt temporarily. During this time the application will no longer respond to user input, giving the impression that the application or the system has crashed. Unsurprisingly this tricks users into thinking that Hermes does not work properly and discourages use.
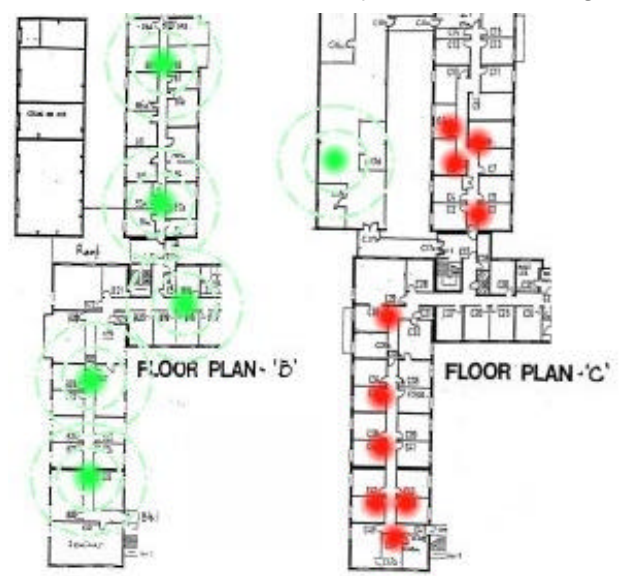

Figure 6: Location of Hermes Displays and 802.11b Access Points

Initially the display cases were completely enclosed aluminium boxes, this design worked well for areas with good signal strength, but in low strength areas the signal was significantly reduced or seemed to be blocked for periods. This problem is aggravated by the fact that the wireless network signal is absorbed by humans. When multiple people gather round a unit (for example to see a demonstration) the network signal can be blocked entirely. Initially it was assumed that the wireless base stations used by the devices were located in the false ceilings of the corridors, though after investigation it was found that all but one of the wireless access points were actually located in the false ceilings of the floor below (this is shown in figure 6, where Hermes displays are shown in red and wireless base stations in green).
This seems to lead to wireless signal 'hot spots' above the access points, though further away the signal strength seems to dictated by the structural anomalies of the building (e.g. Expansion joints between walls significantly reduce signal).

\section{Current solutions}

After experimentation, it was found that removing sections from the top part of the case at the front and back near the protruding part of the wireless network card significantly increased the signal strength. This small modification was so successful the decision was taken to modify all the currently deployed units (as shown in figure 7 ).

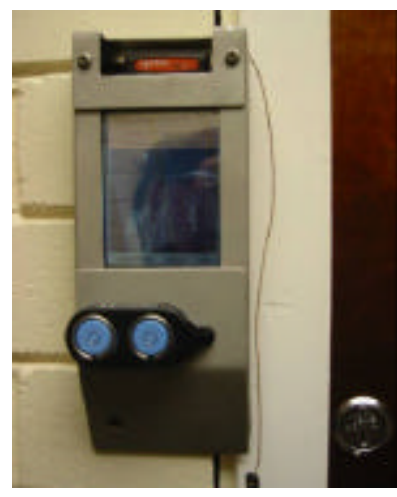

Figure 7: Modified Hermes display housing

We are currently developing a prototype display using a wired ethernet card which we hope will improve users' perceptions of reliability. This has proved challenging due to hardware constraints, the PDA in use, HP Jornada 568, only accepts type I compact flash cards, and type 1 ethernet cards have proved relatively scarce. For various reasons, including the need to utilize existing hardware, our current solution is to use a modified Compaq IPAQ and compact flash expansion jacket with a type 2 wired ethernet card.
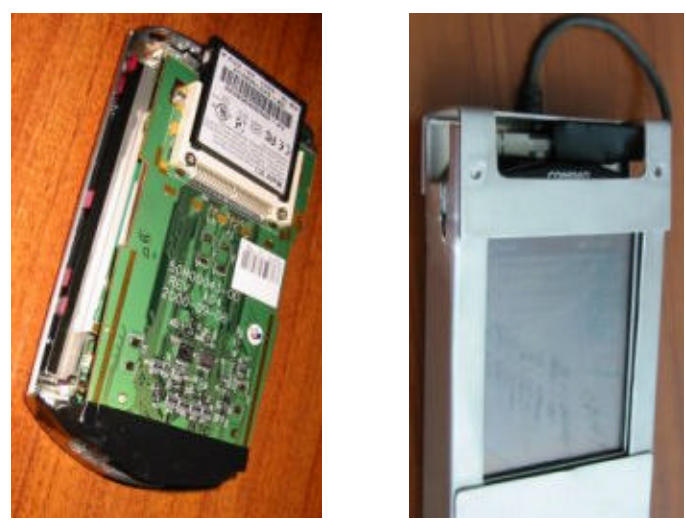

Figure 8: Modified Compaq IPAQ

We found that an IPAQ without the rear casing (but including battery) along with a Compact Flash jacket without any of the casing (see figure 8 - left), would fit in our housing. 
The housing did require slight modifications (as can be seen in figure 8 - right) to accommodate the slight 'bulge' at the top of the iPAQ case. Also, removing the housing from the connector 'dongle' of the ethernet card allowed it to fit in the space designed for the protruding section of a compact flash wireless network card (this can be seen in figure 8 - right). It should be noted that all of the exposed circuit boards and electronic components are entirely insulated before the device is placed in the housing.

Presently there are no viable methods we can use to improve the current hardware and software platforms to provide the high levels of dependability we need. It is simply not possible to change or upgrade all of the Hermes display hardware. Additionally it is not always the hardware at fault; several pieces of $3^{\text {rd }}$ party software (among other things) have proved themselves unreliable. The key to improving dependability and users' perception of dependability may not lie solely in preventing faults. We feel it can be improved through accepting that faults may occur, and providing measures to minimise downtime and inform users of the system status.

Previously we relied on users notifying us when their display had crashed, or through the chance encounter of noticing when one wasn't working. Our present solution is to use a Management Agent to monitor all the displays and provide (among other features) automated notification on display failure.

\section{SUPPORTING AND MANAGING HERMES}

The main motivation for the Management Agent is to provide a system to monitor the Hermes displays, so both system administrators and users can be alerted to display failure. While designing this new system we also took the opportunity to include desirable features we felt would be useful. The following sections explore the important aspects of the Management Agent.

\section{Notification}

One of the key methods we identified to increase a users trust of their Hermes display is providing them with notification of system failure. Initially an e-mail list was set up containing the addresses of all door display owners, this is used to notify users of scheduled system downtime for upgrade work etc. This idea has been extended using the Management Agent to provide e-mail notification on individual Hermes display failure to both the owner of the Hermes display and system administrator. A similarly important feature is that of notifying user when their Hermes display is working again.

This new functionality has only recently been enabled, though it is hoped that this extra information will increase users trust in Hermes, as they will always be aware of whether their Hermes display is working or not. There is also the added advantage that the system administrator learns of a Hermes display failure at the earliest opportunity. Enabling any problems to be resolved quickly and improving overall reliability.

\section{Management Agent Web Portal}

The Management Agent also includes a web portal, accessible by Hermes user with correct privileges. This allows easy access to management features both locally and remotely. Presently there is only a single access level, allowing access to all management features. However this may be extended, allowing subsets of features to users who may need them.

\section{Monitoring}

The main web portal screen is shown in figure 9, and shows the status of the Hermes displays for all users in a table, one row for each user. It shows their username, real name (an e-mail link), office number and the last date and time their Hermes display was know to be alive.

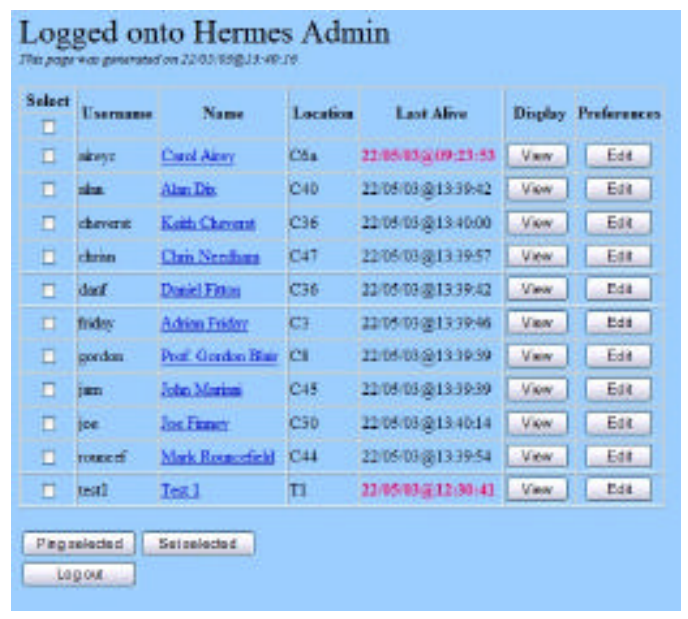

Figure 9: Web portal main screen

If a user's Hermes display has not communicated with the server for a period of time (and assumed to be 'dead') the entry is highlighted in red. Each row in the table provides a 'View' button to see what is currently displayed on the user's Hermes display (figure 10).

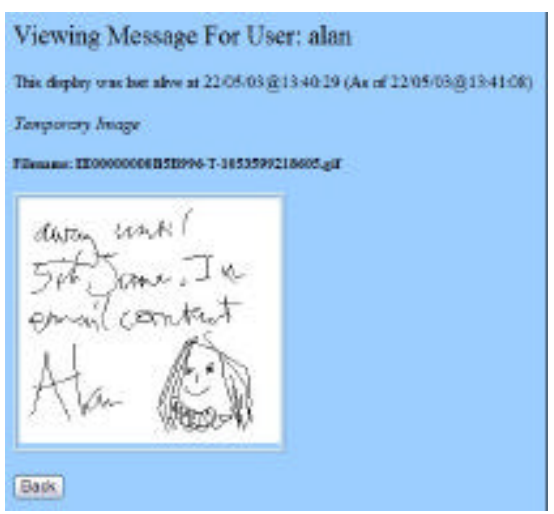

Figure 10: Viewing a user's Hermes display.

A second button is also provided to view and edit users personal preferences. Another important feature 
incorporated is that of 'pinging' a Hermes display, this feature initiates a sequence of remote calls to and from a Hermes display to verify that the Hermes application and wireless network are functioning correctly. If a 'ping' is successful the server has an updated 'last alive' time for that Hermes display, which is reflected in the web portal.

To initiate a 'ping' on a Hermes display the administrator ticks the check box on the appropriate row in the table, then selects the 'Ping Selected' button. This design allows for a simple interface and allows multiple Hermes displays to be 'pinged' simultaneously.

These features allow a system administrator to monitor all Hermes displays from a single point. This is useful for many reasons, such as:

- to check if a Hermes display is actually malfunctioning

- to check where an update has been successful

- to monitor the Hermes system remotely

\section{Additional Administrator Features}

When designing the Management Agent functionality was included allowing a Hermes administrator to change the temporary message for one or more users. When selecting the users to change the temporary message for, this feature is similar in use to pinging Hermes displays (selection using check boxes). When the administrator selects the 'Set Selected' button they are presented with the dialogue shown in figure 11.

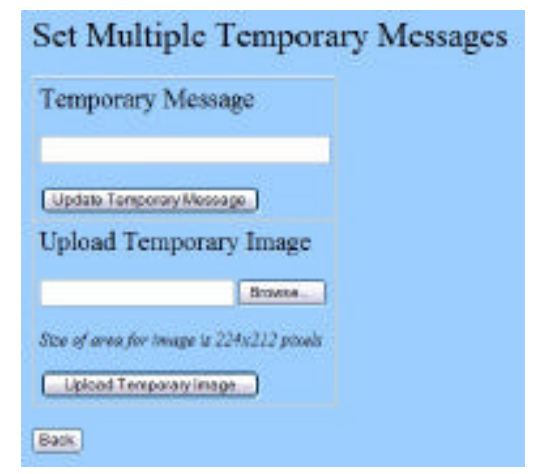

Figure 11: Set temporary messages for multiple users

This allows the administrator to set a textural message, or upload an image file to be used. Once the temporary messages have been changed the administrator may use the 'View' feature (as shown in figure 10) to ensure that displays have been updated successfully. When a user's temporary message has been changed from the Management Agent, they are informed via an e-mail also including reassurance that they can change or remove this message at any time.

We hope to extend this feature so users such as departmental secretaries (etc) may be able to use multiple Hermes displays to display pertinent messages and reminders. These messages may include reminders events such as a fire alarm test, notification of an important meeting, department open days etc.

\section{Current Findings}

The Management Agent has been in place for approximately 10 weeks and although initial signs are encouraging the period of time has not been long enough to justify an analysis of whether it has increased user trust of the system. All use of Hermes is logged so it will certainly be possible to see whether use of Hermes has increased, and additionally to interview users to find their opinions.

The mechanism to detect whether a Hermes display is still working (to provide notification on failure etc) relies on an RMI call back made to the server every minute by the display. A timer is used to detect a problem if the server does not receive any communication from a client for a specified period of time. During development this time was initially set to 5 minutes, though after occasional 'false alarm' notifications of dead displays this was increased to 15 minutes. After deployment it was found this interval was still too short, especially in one area of very low wireless network signal. The Hermes displays could loose signal for around 20 minutes then start working again normally. The timeout was further increased to take this into account, though later the decision was taken to use wired network for that display due to the low signal problem.

The greatest benefit so far from the management agent has been the automated notification of malfunctioning Hermes displays, allowing the problem to be remedied straight away. It is also very for Hermes administrators to be able to monitor the displays and use to ping feature to tell if a display is working immediately.

\section{FUTURE WORK}

Out immediate plans are to provide a new mechanism allowing users (such as departmental secretaries) to set temporary messages for multiple Hermes displays. The issues arsing from the devolvement of this feature will hopefully prove useful, especially as we plan to investigate a departmental navigation system to run on the Hermes displays. The whole area of appropriating a personal public display for other uses enables many appealing applications, and will be interesting to explore.

The central point of control that the Management Agent provides can certainly be exploited through extra features. Providing remote control of the Hermes displays would be very useful, perhaps allowing the Hermes application to be terminated and restarted if it crashed, and allowing updated software versions to be sent to each display. Presently these operations are done manually as all the displays are situated 
relatively close to each other, if they were distributed any further away this may prove a problem. Throughout the Hermes project we have applied a participatory design based approach to development, this will be carried through to the Management Agent as we refine the current design and add new features.

\section{RELATED WORK}

Work has previously been conducted on this specific area at Georgia Tech. However, there work on dynamic door displays [5] appears to have stopped before any significant deployment or reasonable evaluation of the system was able to take place.

McCarthy developed the 'OutCast' service to provide "a personal yet shared display on the outside of an individuals office" [4]. Although OutCast supports a range of features, including a message box feature, it does not support remote interaction and does not allow users to utilise the displays of other office workers. The system is also reported as having only one unit deployed.

Research on utilizing doors as interruption gateways and aesthetic displays is being conducted at CMU [6]. The most similar aspect of this work is its concern for supporting the personalization of information on office doors which it supports in a very rich way through the use of projection systems.

Perhaps the most striking similarity to the motivation and ideas of the Hermes system is the concept of 'infoDoors' described by Ben Schneiderman [8]. In common with our work, Schneiderman envisions buildings with displays outside every office and supporting a variety of uses such as displaying personal messages.

\section{CONCLUDING REMARKS}

This paper has described our experiences of managing and maintaining the Hermes system, a system of deployed office door displays which provide asynchronous messaging services for users in the computing department of Lancaster University. We believe that, in general, the management and maintenance of deployed ubicomp systems is a critically under researched area and we hope that some of the issues and anecdotes presented in this paper and based 15 months experience deploying and maintaining the Hermes system will help to contribute some useful knowledge in this area.

\section{ACKNOWLEDGMENTS}

The work described in this paper has been conducted through the auspices of the CASCO project which is funded by the UK Engineering and Physical Sciences Research Council. We would also like to acknowledge the support of members of Lancaster University's computing department.

\section{REFERENCES}

1. Cheverst, K. , Fitton, D. , and Dix A. "Exploring The Evolution Of Office Door Displays", In K. O'Hara, M. Perry, E. Churchill, D. Russell (ed) Public and Situated Displays: Social and Interactional aspects of shared display technologies. To appear.

2. Cheverst, K. , Fitton, D. , Dix, A. , and Rouncefield, M. "Exploring the use of Remote Messaging and Situated Displays", In Proc of Fifth International Symposium on Human Computer Interaction with Mobile Devices and Services (MobileHCl '03). To Appear.

3. iButton home page: http://www.ibutton.com/

4. McCarthy, J. , Costa, T. , and Liongosari, E. "UniCast, OutCast \& GroupCast: An Exploration of New Interaction Paradigms for Ubiquitous, Peripheral Displays", Workshop on Distributed and Disappearing User Interfaces in Ubiquitous Computing at CHI 2001 (2001).

5. Nguyen, D. , Tullio, J. , Drewes, T. , and Mynatt, E. "Dynamic Door Displays." Unpublished, Georgia Tech: http://www.cc.gatech.edu/fce/ ecl/projects/drewes/DynDoorDisplays.pdf

6. Nichols, J. , Wobbrock, J. , Gergle, D. , and Forlizzi, J. "Mediator and Medium: Doors as Interruption Gateways and Aesthetic Displays" In Proc. of DIS'2002, London, UK. June 25-28. pp. 379-386. (2002)

7. NSiCom CrEme home page: http://www.nsicom.com/

8. Schneiderman, B. "Leonardo's laptop: Human Needs and the New Computing Technologies", MIT Press, ISBN: 0-262-19476-7 (2002)

9. Weiser, M. "The Computer for the 21st Centuary", Scientific American, Vol. 265 No. 3, pp. 66-75. (1991) 